Reprod. Nutr. Dévelop., 1981, 21 (5A), 671-680.

\title{
Action féminisante de la déhydroépiandrostérone sur le testicule gauche de l'embryon de poulet : aspect ultrastructural
}

\author{
par A. PETIT, J. THIEBOLD
}

Laboroloire de Zoologie et d'Embryologie expérimentale de I'Université Louis-Pasteur, 12, rue de l'Université, 67000 Strasbourg.

Summary. Feminizing action of dehydroepiandrosterone on the left festis of the chick embryo : ultrastructural aspect.

Chick embryos were injected with $4 \times 0.5 \mathrm{mg}$ of dehydroepiandrosterone from day 4 onwards and the ultrastructure of the 20-day old hormone-treated male gonads was studied. The cortex was well-developed and some oocytes had reached the meiotic prophase stage. The prefollicular cells did not seem to be stimulated by the androgenic hormone. We especially studied the seminiferous cords with Sertoli cells. Dense core granules, which might be related to secretory activity, were observed in the basal part of the Sertoli cells. The extensive development of smooth endoplasmic reticulum vesicles suggested that some Sertoli cells might have a steroidogenic function. The interstitial cells did not show an important steroidogenic synthesis. The action of dehydroepiandrosterone, which could induce the development of an ovarian-like cortex in intersexual gonads, is discussed.

\section{Introduction.}

Comme les œstrogènes, certains androgènes, administrés à l'embryon de poulet, féminisent les mâles génétiques en induisant, en particulier, un développement cortical dans la gonade gauche (Wolff et al., 1948 ; Weniger et Zeis, 1973a). L'inversion du sexe par les hormones stéroïdes relève d'un mécanisme mal connu et les observations ultrastructurales qui s'y rapportent sont encore très limitées (Narbaitz, 1971 ; Carlon et Erickson, 1978).

L'hormone agit-elle directement sur les cellules germinales ou bien par l'intermédiaire des cellules préfolliculeuses? Quels sont les rapports entre ces deux types cellulaires? Les cellules de Sertoli des cordons séminifères sont-elles stimulées par l'androgène et, dans l'affirmative, présentent-elles des signes d'activité stéroïdogène ? En l'absence de canaux de Müller chez les mâles féminisés, les cellules de Sertoli manifestent-elles une activité particulière qui pourraił traduire l'existence de l'hormone anti-müllérienne? Les cellules interstitielles sont-elles différenciées ? Dans cette étude, nous essayerons de répondre à ces questions.

Le présent travail se rapporte à l'action féminisante envisagée à l'échelon ultrastructural, d'une hormone androgène, la déhydroépiandrostérone dont seul le métabolisme a fait l'objet de recherches antérieures (Haffen et Cedard, 1968). 


\section{Matériel et méthodes.}

Nous avons utilisé dans cette étude 35 embryons de poulet (Gallus gallus L., race Leghorn blanche) : 25 embryons ont reçu chacun $500 \mu \mathrm{g}$ de $\mathrm{DHA}$ ( $\Delta^{5}$-andro-stène-3 $\beta$ 01-17-one) dissous dans $20 \mu$ l d'huile d'olive neutralisée, aux $4 \mathrm{e}, 7 \mathrm{e}, 10^{\mathrm{e}}$ ef $13^{\mathrm{e}}$ jours de l'incubation et 10 embryons témoins ont reçu parallèlement $20 \mu l$ d'huile d'olive. La DHA (l'Industrie biologique française) a d'abord été dissoute dans une petite quantité d'éther avant d'être ajoutée à l'huile d'olive, l'éther étant ensuite éliminé à l'étuve à $40^{\circ} \mathrm{C}$. La solution hormonale a été simplement déposée sur la membrane coquillière de la chambre à air de l'œuf. Les 14 embryons traités et vivants ont été sacrifiés au $20^{e}$ jour de l'incubation ainsi que les 10 témoins. Les gonades, découpées en fragments, ont été fixées pendant $20 \mathrm{~min}$ à $4^{\circ} \mathrm{C}$ dans le glutaraldéhyde à 3 p. 100 dans le tampon phosphate $0,1 \mathrm{M}$ à pH 7,4 selon Sörensen. Après 3 rinçages dans le tampon, les pièces postfixées dans le tétroxyde d'osmium à 2 p. 100 pendant $1 \mathrm{~h}$ à $4^{\circ} \mathrm{C}$, ont été incluses dans l'araldite puis débitées en coupes semi-fines et ultrafines à l'ultrotome PorterBlum MT2. Les coupes semi-fines ont été colorées au bleu de méthylène-Azur II (Richardson, 1960). Les coupes ultrafines, contrastées à l'acétate d'uranyle-citrate de plomb (Reynolds, 1963) ont été examinées à l'Elmiscop IA Siemens.

\section{Observations.}

Les embryons traités el vivants au 200 jour se répartissent en 8 mâles féminisés et 6 femelles d'apparence normale. Macroscopiquement, les intersexués se reconnaissent aisément : la gonade gauche aplatie est nettement plus volumineuse que la gonade droite cylindrique ; les canaux de Müller sont absents comme chez les mâles témoins.

Les coupes semi-fines (fig. 2) mettent en évidence, dans tous les cas, un cortex développé pouvant représenter le tiers de l'épaisseur de l'organe, comme c'est habituellement le cas chez les femelles témoins. La région médullaire comprend des cordons séminifères, des cellules interstitielles groupées en îlots, des vaisseaux sanguins ef du mésenchyme lâche. Des lacunes en nombre et dimensions très variables parsèment le territoire médullaire.

\section{PLANCHE I}

FIG. 1. - Coupe dans le corfex d'un ovotestis. Présence d'un pont cytoplasmique (P) entre deux ovocytes (1) dont l'un entre en prophase méiotique. Les mitochondries se groupent autour des éléments golgiens $(G)$ pour former le corps vitellin de Balbiani. Les cellules préfolliculeuses ont des prolongements cytoplasmiques $(2) . \times 14500$.

FIG. 2. - Coupe semi-fine dans un ovotestis. La zone corticale (C) est très épaisse. Dans la zone médullaire $(M)$ se trouvent les cordons séminifères. $\times 160$.

FIG. 3. - Coupe dans le cortex d'un ovotestis. L'ovocyte (1), au stade zygotène, possède des complexes synaptonématiques $(S)$. Les pores nucléaires sont nombreux. Le corps vitellin de Balbiani (B) est localisé à un pôle de l'ovocyte. Les cellules préfolliculeuses (2) tendent à entourer l'ovocyte de leurs expansions cytoplasmiques. $\times 14000$. 
Le corfex : Sous un épithélium formé d'une ou parfois de deux assises cellulaires, les cellules germinales sont groupées en massifs faisant saillie dans la zone médullaire. Ces massifs sont séparés par des travées de cellules mésenchymateuses étroites et très allongées. Les éléments germinaux sont des ovogonies et des ovocytes. Les ovogonies en proportion plus grande que les ovocytes ont un noyau arrondi dont l'hétérochromatine est régulièrement répartie. Le nucléole est formé d'une ou de deux masses denses souvent alvéolées. Le nombre important de pores nucléaires caractérise toutes les cellules germinales (fig. 1 et 3 ). Des ovocytes en préméiose (fig. 3) se rencontrent dans le cortex de tous les ovotestis. Le cytoplasme des ovogonies et des ovocytes est moins dense que celui des cellules préfolliculeuses (fig. 3). Il contient un corps vitellin de Balbiani (fig. 3) moins riche en vésicules et en mitochondries que celui des ovocytes des ovaires témoins. Des cellules germinales en nécrose parsèment le cortex.

Les cellules préfolliculeuses ont un noyau irrégulier, cerné de petits amas d'hétérochromatine. Leur cytoplasme dense (fig. 3) renferme de nombreux ribosomes libres, peu de vésicules du réticulum lisse ou granulaire et peu d'inclusions lipidiques. Au $20^{e}$ jour de l'incubation, les cellules préfolliculeuses ne manifestent pas encore de tendance à former des follicules ; souvent, leurs expansions cytoplasmiques longues et étroites traversent des lacunes avant d'établir un contact avec les ovocytes. Nous n'avons pas rencontré les structures particulières, issues de la membrane plasmique des cellules préfolliculeuses des ovaires embryonnaires, appelées «lining bodies» (Bellairs, 1965). Comparé aux témoins, le cortex des ovotestis a une structure quelque peu lacunaire ; les cellules germinales peuvent entrer en prémélose, mais la plupart d'entre elles, cependant, sont au stade d'ovogonies; les cellules préfolliculeuses ne semblent pas activées par l'androgène et ne possèdent pas de «lining bodies ».

La région médullaire : Les cordons séminifères apparaissent comme des massifs cellulaires allongés ou contournés, toujours limités par une basale. A la différence de ce qui s'observe dans les gonades mâles de même âge, l'organisation fubulaire avec ébauche de lumière centrale n'est jamais réalisée. Les cellules germinales ou sperma togonies, claires, peu nombreuses, se distinguent des cellules de Sertoli plus denses aux électrons et plus abondantes. Leur grand axe est très souvent perpendiculaire à la basale (fig. 6). Leur partie apicale contient un noyau arrondi où, parmi l'hétéro-

\section{PLANCHE \|}

FIG. 4. - Coupe dans des cellules de Sertoli d'un cordon séminifère d'ovotestis. Noter le noyau échancré caractéristique à position basale et le nucléole $(N)$ à plage centrale plus claire. Des grains de sécrétion $(G)$ sont identifiables. La membrane plasmique contiguë à la basale (B) montre des vacuoles de pinocytose $(\mathrm{PI}) . \times 15000$.

FIG. 5. - Coupe dans une cellule de Sertoli d'un ovotestis. Les grains de sécrétion à cœur dense s'accumulent vers le pôle basal (B) de la cellule. $\times 26000$.

FIG. 6. - Coupe dans un cordon séminifère d'ovotesfis. Les cellules germinales (1) sont plus claires que les cellules de Sertoli (2). Les pores nucléaires (PN) des spermatogonies sonf nombreux. La cellule germinale et la cellule de Sertoli ont leur grand axe perpendiculaire à la basale : le noyau germinal est en position apicale, le noyau sertolien en position basale. (GS), grain de sécrétion. $\times 12000$. 


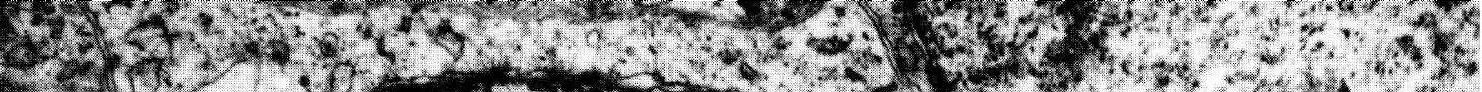

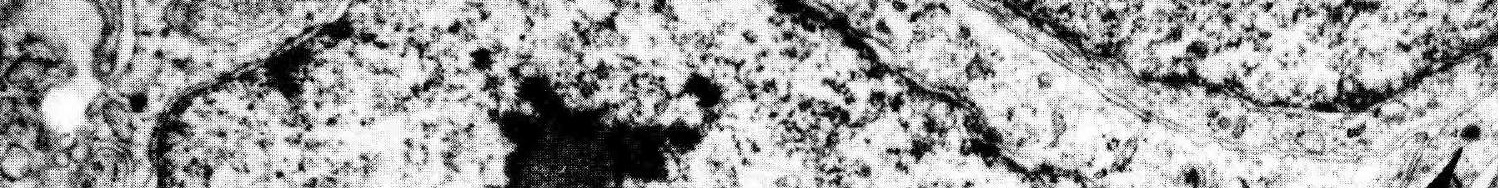

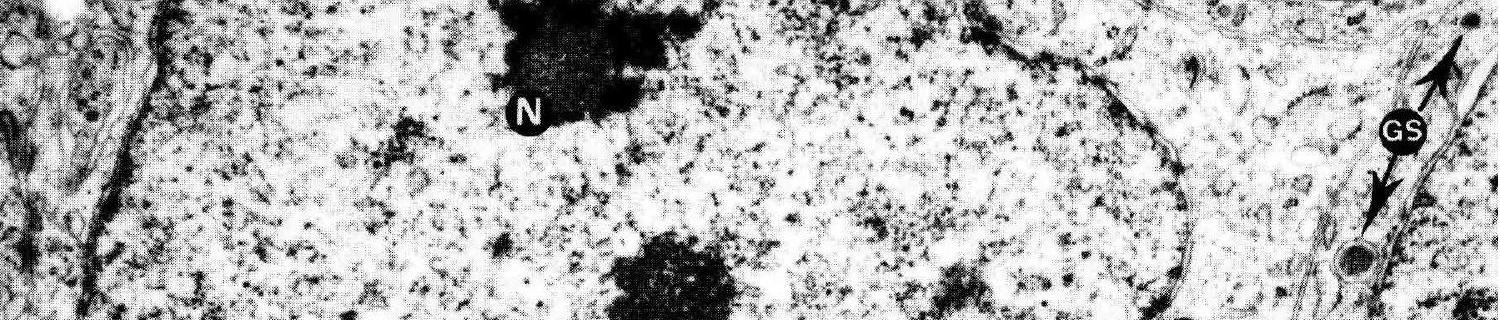
7,5

$x+4$

$x+2$

.

wh.

x





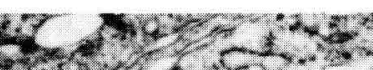

27

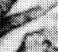

0765

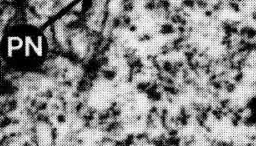

ancto $+2,0,+4$ 西

x. $x$

(4) $4 \times$ f $x$, s.

a. 0. 0.5



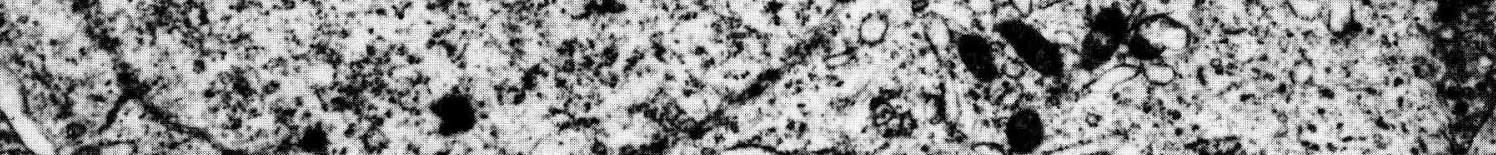

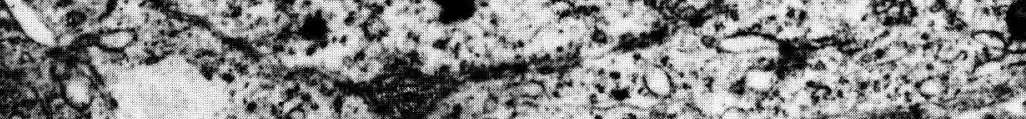


chromatine régulièrement répartie, se distingue un nucléole fait de plusieurs masses denses finement granulaires (fig. 6). Les spermatogonies des ovotestis ressemblent à celles des testicules témoins.

Les cellules de Sertoli, généralement allongées, n'ont pas une orientation préférentielle aussi marquée que les spermałogonies. Leur noyau est très souvent échancré (fig. 4). Lorsque la cellule est allongée perpendiculairement à la basale, le noyau est situé près de cette dernière. Le nucléole, finement granulaire, renferme souvent une plage centrale plus claire (fig. 4). Leur cytoplasme apparaît modérément dense ou très dense ; il possède des mitochondries à crêtes transversales, des rubans ergastoplasmiques, des ribosomes parfois groupés en rosettes, des vésicules du réticulum lisse, éventuellement quelques lysosomes eł presque toujours des granules denses d'origine golgienne (fig. 4). Ces granules ont une nette polarité basale (fig. 5) et sont formés d'une vésicule $(1500 \AA<\varnothing<3700 \AA)$ contenant un grain dense $(500 \AA<\varnothing<3000 \AA)$. La membrane plasmique contiguë à la basale révèle des phénomènes de pinocytose (fig. 4). Les vésicules réticulaires lisses sont en nombre très variable, mais certaines cellules en renferment une grande quantité (fig. 7). Des inclusions lipidiques se trouvent assez souvent parmi les vésicules réticulaires (fig. 7). D'après la forme du noyau et des mitochondries ainsi que par la présence de vésicules à cœur dense, nous identifierons ces cellules comme étant des cellules de Sertoli. Dans les tubes séminifères des testicules de même âge, nous n'avons pas rencontré de cellules de Sertoli présentant les indices d'une activité stéroïdogène aussi marquée.

Les cellules interstitielles, aisément identifiables, sont groupées par trois ou quatre dans les espaces intercordonnaires ou interlacunaires. Elles sont caractérisées par des mitochondries à crêtes fubulaires, des citernes du réticulum lisse, des polysomes et des inclusions lipidiques souvent volumineuses (fig. 8). Il existe des cellules interstitielles à cyłoplasme dense et des cellules interstitielles à cyloplasme clair (fig. 8). Ces dernières, les moins nombreuses, ne contiennent pas d'inclusions lipidiques, ont moins de ribosomes et représentent probablement une phase plus active que les premières (Russo ef al., 1971). Dans les festicules témoins, nous n'avons rencontré que des cellules interstitielles denses ayant des inclusions lipidiques.

PLANCHE III

FIG. 7. - Coupe dons des cellules de Sertali d'un ovotestis. Le caractère stéroïdogène est mis en évidence par la concentration importante de vésicules du réticulum lisse (V). Parmi les vésicules se trouvent de petites inclusions lipidiques $(L)$ à contour anguleux. Des mitochondries $(M)$ sont au voisinage des vésicules. (E), interdigitation. (D), desmosome. $\times 20000$.

FIG. 8. - Coupe dans le stroma médullaire d'un ovofestis. Les cellules interstitielles denses (1) renferment des ribosomes, des mitochondries $(M)$ à crêtes devenant tubulaires, des inclusions lipidiques de grande taille $(L)$ et des vésicules réticulaires lisses (V). Les cellules interstitielles claires (2) ont moins de ribosomes et pas de grosses inclusions lipidiques. Les cellules mésenchymateuses (3) sont très allongées. $\times 13000$. 


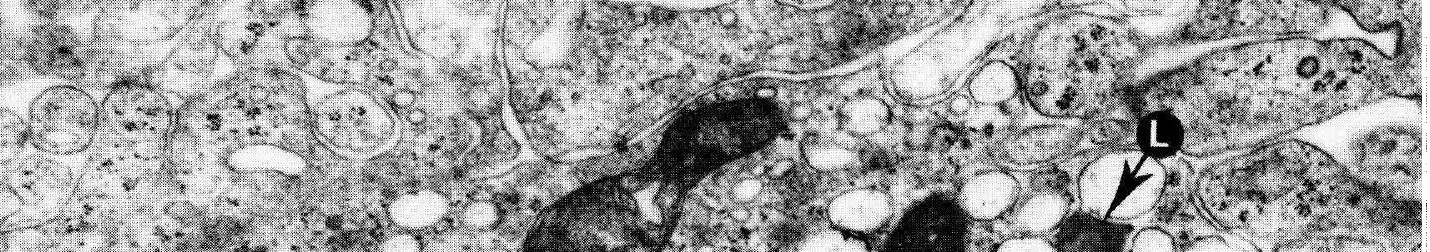

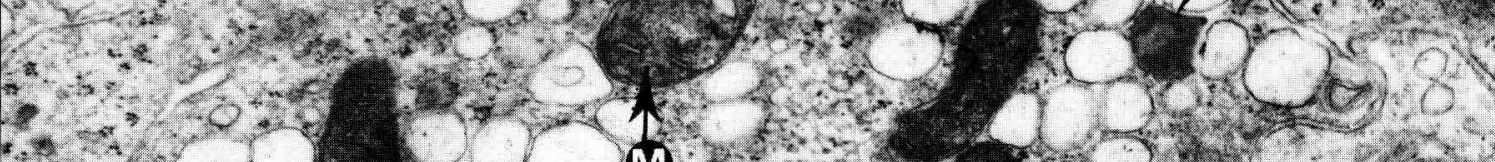 (1)




C.2.

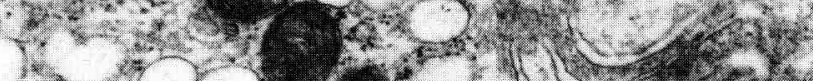



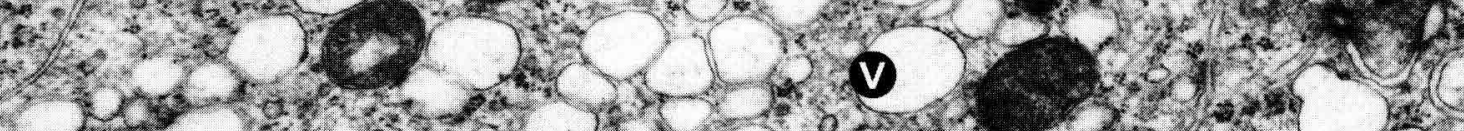

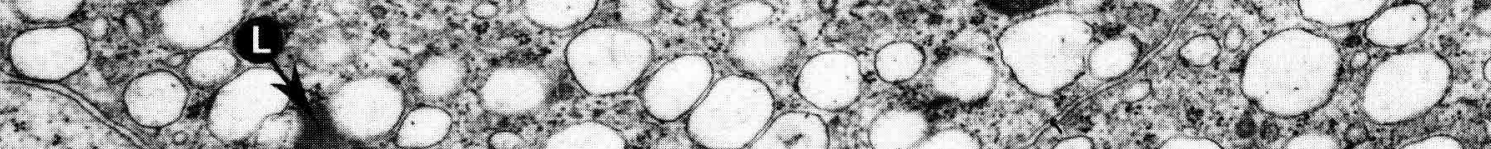

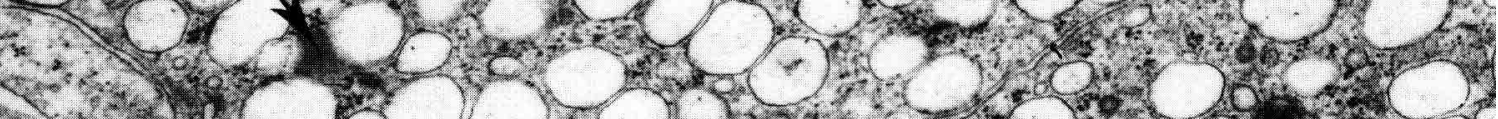
4.

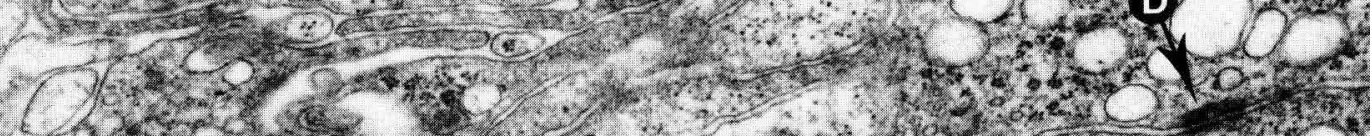

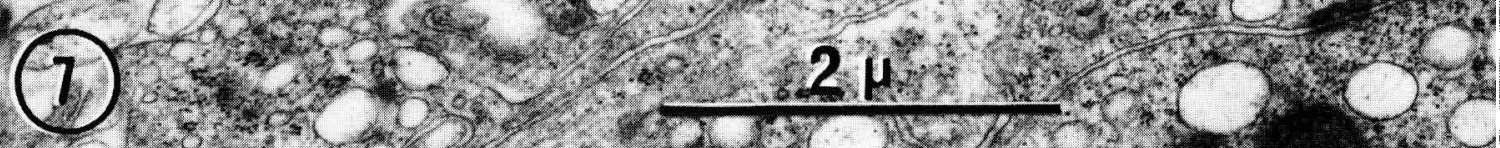
Lis.

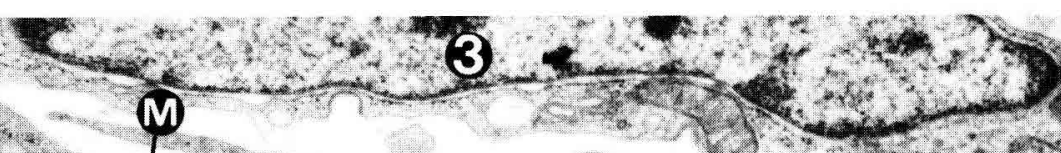

$$
\begin{aligned}
& \text { Q }
\end{aligned}
$$

6.2.

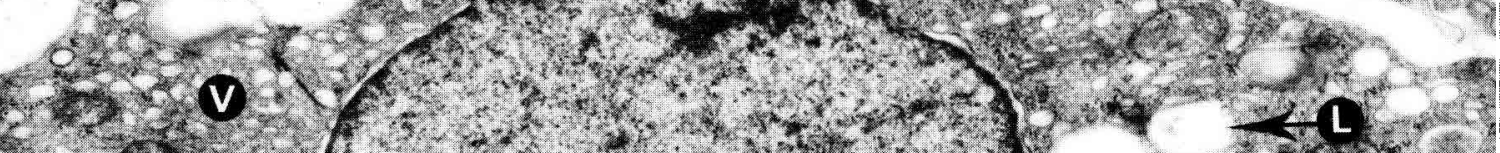

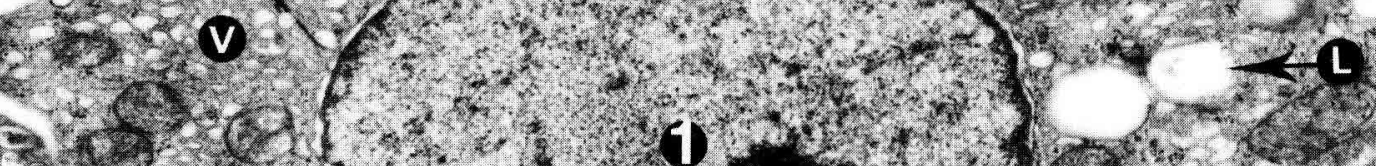

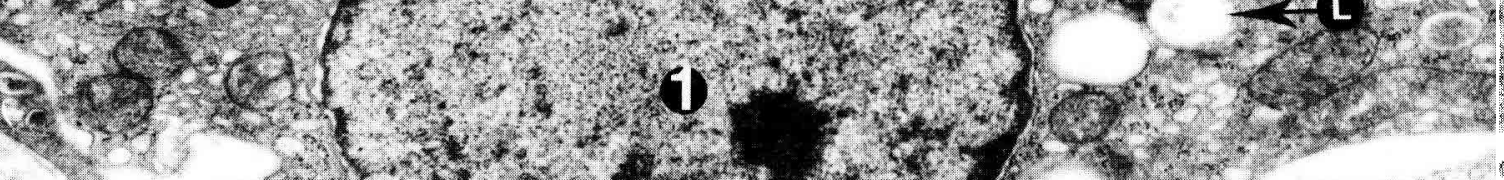

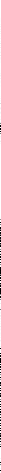




\section{Discussion.}

La DHA féminise le testicule gauche de l'embryon de poulet. Le cortex de l'ovotestis présente des ovocytes en prophase méiotique. Ces résultats sont comparables à ceux obtenus par Narbaitz (1971) avec le benzoate d'œstradiol. Par contre, Carlon et Erickson (1978) en utilisant l'androstènedione et la dihydrotestostérone obtiennent un cortex uniquement fait d'ovogonies, alors que les ovocytes des ovaires témoins sont en prophase. Ces auteurs, qui ont réalisé leurs expériences in viłro, ont utilisé de faibles quantités d'androgènes. Le déclenchement de la prophase méiotique que nous avons obfenu pourrait être dû à la forte dose de DHA utilisée au cours d'injections répétées.

La DHA induit la différenciation des cellules préfolliculeuses. Par comparaison avec les témoins, il faut noter l'absence de « lining bodies » qui sont observés après utilisation du benzoate d'œestradiol (Narbaitz, 1971; Carlon et Erickson, 1978), de l'androstènedione et de la dihydrotestostérone (Carlon et Erickson, 1978). La DHA ne provoque pas une activation particulière et visible au microscope électronique des cellules préfolliculeuses comme c'est le cas après l'action de la dihydrotestostérone (Carlon et Erickson, 1978); de nombreuses citernes ergastoplasmiques traduisent alors une synthèse active, probablement protéique selon ces auteurs. L'hyperactivité sécrétoire, qui n'existe d'ailleurs pas chez les témoins, doit être liée à la nature de l'hormone utilisée.

Les cellules de Sertoli des testicules féminisés ont beaucoup moins retenu l'attention des auteurs. Pourtant, la présente étude montre que leur différenciation est aussi avancée que dans les testicules témoins. L'existence de citernes ergastoplasmiques suggère une synthèse protéique active dont les indices ont également été observés dans les cellules de Sertoli de l'embryon de rat (Merchant-Larios, 1976 ; Hatier et Grignon, 1980).

La présence d'un grand nombre de vésicules réticulaires lisses et d'inclusions lipidiques est l'indice d'une activité stéroïdogène dans certaines cellules somatiques des cordons séminifères des ovotestis. Ayant observé une série d'intermédiaires entre la cellule de Sertoli et la cellule à caractère stéroïdogène, nous admettons que la dernière dérive de la première. Par ailleurs, les cellules de Sertoli sont reconnues aptes à synthétiser des stéroïdes (de Martino et al., 1977; Tcholakian et Steinberger, 1978).

L'activité sécrétoire sertolienne, qui se manifeste par des grains à cœur dense à polarité basale, mérite d'être soulignée. Hatier et Grignon (1980) observent également ces granules dans les cellules de Sertoli du testicule embryonnaire du rat. Chez les intersexués que nous avons observés, les canaux de Müller sont absents alors que les femelles, bien que traitées par la DHA, ont des canaux typiques. Par conséquent, les cellules de Sertoli des ovotestis ont été aptes à synthétiser l'hormone anti-müllérienne. L'existence de cette hormone de nature glycoprotéique a déjà été révélée, chez les Mammifères, dans ce type cellulaire (Josso et al., 1977 ; Picard et al., 1978). Comple tenu de l'activité anti-müllérienne encore importante au $18^{\mathrm{e}}$ jour de l'incubation (Maraud ef al., 1966), il est permis d'envisager, comme hypothèse de travail, que les grains de sécrétion traduisent la présence de l'hormone au $20^{\mathrm{e}}$ jour aussi bien chez les intersexués que chez les mâles témoins.

Les cellules interstitielles des ovotestis ont toutes les caractéristiques de cellules stéroïdogènes mais ne semblent pas dans une phase très active en raison du nombre 
assez faible de vésicules réticulaires lisses. Les cellules claires pourraient représenter un stade intermédiaire entre les cellules de Leydig et les cellules de Sertoli stéroïdogènes et apporter ainsi un argument en faveur de l'origine cordonnaire des cellules interstitielles (Scheib, 1970).

Comment la DHA peut-elle induire le développement d'un cortex dans le testicule gauche? Une étude ultrastructurale ne permet pas de répondre à cette question mais nous pouvons cependant aborder ce problème difficile. Les ovotestis sont capables d'aromatiser la DHA en œstrogènes (Haffen et Cedard, 1968). Le testicule féminisé par la dihydrotestostérone produit des œstrogènes (Weniger et Zeis, 1973b). II s'ensuit que la féminisation par un androgène paraît moins paradoxale, mais l'existence des enzymes de l'aromatisation avant le développement cortical est un point encore obscur. La mise en évidence de sites récepteurs à l'œstradiol dans l'épithélium germinatif d'embryons mâles et femelles de $51 / 2$ à 7 jours (Gasc, 1980) permet d'entrevoir le mode d'action des œestrogènes. Les cellules mésothéliales de l'épithélium germinatif qui possèdent probablement ces sites, se différencieraient sous l'action hormonale exogène en cellules préfolliculeuses qui, à leur tour, agiraient sur les cellules germinales, permettant ainsi le développement d'un cortex. D'après nos résultats, la présence d'un cortex n'inhibe pas ou ne retarde pas la différenciation des cellules de Sertoli dans les cordons séminifères, l'absence des canaux de Müller et les figures de stéroïdogenèse en sont la preuve. La présence simultanée d'éléments mâles et femelles dans la gonade gauche de Poulet à la suife de l'action d'une hormone mâle, rend bien compte de la complexité des phénomènes qui régissent la différenciation sexuelle.

\section{Conclusion.}

L'éfude ultrastructurale du testicule de poulet féminisé in vivo par la DHA a apporté les principaux résultats suivants :

- la DHA induit un cortex où les ovocytes peuvent entrer en prophase méiotique :

- les cellules préfolliculeuses sont différenciées mais n'ont pas une activité métabolique particulière due à l'hormone injectée :

- des cellules de Sertoli présentent des signes de stéroïdogenèse active ;

- l'activité sécrétoire des cellules de Sertoli est aftestée par la présence de granules denses à polarité basale, probablement en rapport avec la synthèse de l'hormone antimüllérienne ;

- les cellules interstitielles sonł différenciées.

Reçu en jonvier 1981.

Accepté en mors 1981.

\section{Références}

AKRAM H., WENIGER 3. P., 1967. Sécrétion d'cestrone et d'cestradiol par le testicule féminisé de l'embryon de poulet. C. R. Acad. Sci. Paris, 264, 1806-1807.

BELLAIRS R., 1965. The relationship between oocyte and follicle in the hen's ovary as shown by electron microscopy. J. Embryol. exp. Morph., 13, 215-233. 
CARLON N., ERICKSON G. F., 1978. Fine structure of prefollicular and developing germ cells in the male and female left embryonic chick gonads in vitro with and without androgenic steroids. Ann. Biol. anim. Bioch. Biophys., 18, 335-349.

GASC J. M., 1980. Estrogen target cells in gonads of the chicken embryo during sexual differentiation. J. Embryol. exp. Morph., 55, 331-342.

HAFFEN K., CEDARD L., 1968. Ełude, en culture organotypique in vitro, du métabolisme de la déhydroépiandrostérone et de la testostérone radioactives, par les gonades normales et intersexuées de l'embryon de poulet. Gen. comp. Endocrinol., 11, 220-234.

HATIER R., GRIGNON G., 1980. Ultrastructural study of Sertoli cells in rat seminiferous tubules during intrauterine life and the postnatal period. Anat. Embryol., 160, 11-27.

JOSSO N., PICARD J. Y., TRAN D., 1977. The antimüllerian hormone. Rec. Progr. Horm. Res., 33, 117-167.

MARAUD R., STOLL R., COULAUD H., 1966. Action de la greffe testiculaire sur les canaux de Müller de l'embryon de poulet. C. R. Soc. Biol., 160, 964-966.

MARTINO C. de, MARCANTE M. L., FLORIDI A., CITRO G., BELLOCI M., CANTAFORA A., NATALIP. G., 1977. Sertoli cells of adult rat in vitro. Cell Tissue Res., 176, 69-90.

MERCHANT-LARIOS H., 1976. The onset of testicular differentiation in the rat : an ultrastructural study. Am. J. Anat., 145, 319-330.

NARBAITZ R., 1971. Ultrastructural aspects of cortical differentiation in chick ovaries and intersexual gonads. Z. Zellforsch., 118, 315-325.

PICARD J. Y., TRAN D., JOSSO N., 1978. Biosynthesis of labelled anti-müllerian hormone by fetal testes : evidence for the glycoprotein nature of the hormone and for its disulfide-bonded structure. Mol. cell Endocrinol., 12, 17-30.

REYNOLDS E. S., 1963. The use of lead citrate at high $\mathrm{pH}$ as an opaque stain in electron microscopy. J. Cell Biol., 17, 208-212,

RICHARDSON K. C., JARETT L., FINKE E. H., 1960. Embedding in epoxy resins for ultrathin sectioning in electron microscopy. Stain Technol., 35, 313-323.

RUSSO J., de RISAS J. C., 1971. Differentiation of the Leydig cell of the mouse testis during the fetal period. An ultrastructural study. Am. J. Anat., 130, 461-480.

SCHEIB D., 1970. Sur la présence de cellules « interstitielles primaires " dans les cordons du testicule de l'embryon de poulet. C. R. Acad. Sci. Paris, Sér. D, 270, 123-125.

TCHOLAKIAN R. K., STEINBERGER A., 1978. Progesterone metabolism by cultured Sertoli cells. Endocrinology, 103, 1335-1343.

WENIGER J. P., ZEIS A., 1973a. Action féminisante des androgènes sur le testicule et le canal de Müller d'embryon de poulet in vitro. Arch. Anat. micr. Morph. exp., 62, 145-150.

WENIGER J. P., ZEIS A., 1973b. Sécrétion d'une hormone féminisante induite dans le testicule embryonnaire de poulet par la dihydrotestostérone. Arch. Anat. micros., 62, 173-176.

WOLFF Et., STRUDEL G., WOLFF Em., 1948. L'action des hormones androgènes sur la différenciation sexuelle des embryons de poulet. Arch. Anat. Hist. Embr., 31, 237-310. 